\title{
Factors Related to Low Back Pain Severity in Veterans with Unilateral Below Knee Amputation
}

\section{ART ICLE INF O}

\section{Article Type}

Descriptive Study

\section{Authors}

Faraji E. ${ }^{1} P h D$,

Barati A.H. ${ }^{2} P h D$,

Sadeghi H. ${ }^{1} P h D$

Fateh H.R. ${ }^{3} M D$,

Allami M.*BSC

How to cite this article
Faraji E, Barati A H, Sadeghi H,
Fateh H R, Allami M. Factors Rel-
ated to Low Back Pain Severity in
Veterans with Unilateral Bel-ow
Knee Amputation. Iranian Jour-
nal of War \& Public Health. 2017
;9(4):185-190.

*Department of Medical \& Rehabilitation Devices, Janbazan Medical and Engineering Research Center (JMERC), Tehran, Iran

${ }^{1}$ Sport Injuries \& Corrective Exercise Department, Physical Education and Sport Sciences Faculty, Kharazmi University, Tehran, Iran

${ }^{2}$ Sport Injuries \& Corrective Exercise Department, Physical Education \& Sport Sciences Faculty, Shahid Beheshti University, Tehran, Iran

${ }^{3}$ Physical Medicine \& Rehabilitation Department, Medicine Faculty, Tehran University, Tehran, Iran

\section{Correspondence}

Address: Janbazan Medical and Engineering Research Center (JMERC), No. 17, Farokh Street, Moghadas Ardabili Street, Yaman Street, Shahid Chamran Highway, Tehran, Iran Phone: +98 (21) 22172424

Fax: +98 (21) 22418180 iranassistive@gmail.com

\section{Article History}

Received: April 12, 2017

Accepted: May 23, 2017

ePublished: November 6, 2017

\section{A B S T R A C T}

Aims Low back pain as a common health issue is more problematic than other complications of amputation. Due to the etiology of amputation in veterans, different factors may be related to the severity of back pain. The aim of this study was to evaluate the factors related to the severity of low back pain in veterans with unilateral below-knee amputation.

Instruments \& Methods In this descriptive correlational study, 62 male veterans with unilateral below-knee amputation in 2016 were selected using available sampling method from Center of Orthosis and Prosthetics Kowsar in Tehran. The severity of back pain and disability caused by low back pain were evaluated by Numeric Pain Rating Scale (NPRS) and Oswestry Disability Index (ODI), respectively. Biomechanical evaluations were carried out using surface electromyography. Data was analyzed by SPSS 20 software using Pearson correlation coefficient, Spearman's rank correlation coefficient, simple linear regression and Chi square test.

Findings There was a significant correlation between pain severity and asymmetry index of superficial lumbar multifidus muscle $(r=0.31 ; \mathrm{p}=0.01)$. Asymmetry index of superficial lumbar multifidus muscle could predict 0.09 of pain severity $(\mathrm{p}<0.05)$. The severity of low back pain were also correlated significantly with smoking history $(r=0.21 ; p=0.04)$, psychological disorders $(r=0.25 ; p=0.02)$, prosthetic height $(r=0.21 ; p=0.04)$ and the use of auxiliary device $(\mathrm{r}=0.03 ; \mathrm{p}=0.27)$.

Conclusion Muscle asymmetry due to the use of the prosthesis, prosthesis height, amputationassociated psychological disorders and smoking are the factors related to the severity of low back pain in veterans with unilateral below-knee amputation.

Keywords Low Back Pain; Veterans; Amputees; Electromyography

\section{I T A T I O N L I N KS}

[1] The epidemiology of low back ... [2] Review of secondary physical conditions associated ... [3] Phantom limb, residual limb, and back pain after ... [4] Phantom pain, residual limb pain, and back pain ... [5] Back pain as a secondary disability ... [6] Pain site and the effects of amputation ... [7] Risk factors for work-related musculoskeletal ... [8] Perceptions of low back pain in people with lower limb ... [9] Diagnosis and treatment of low back ... [10] Systematic review of psychosocial factors at work ... [11] A systematic review of psychological factors as ... [12] Compensatory mechanisms in below-knee [13] Three-dimensional knee joint contact forces ... [14] The influence of increasing [15] Chronic low back pain in traumatic ... [16] When is cancer pain mild, moderate [17] The Oswestry low back pain disability ... [18] The validation of visual analogue ... [19] The Oswestry disability index ... [20] Use of the surface EMG signal ... [21] Surface electromyography ... [22] Lumbar muscle electromyographic ... [23] Endurance of trunk muscles in ... [24] Do exercise balls provide ... [25] Electromyographic activity of the ... [26] Different ways to balance the ... [27] Motor training of the lumbar ... [28] Intensive unilateral neuromuscular ... [29] A preliminary investigation ... [30] Adaptation strategies of the ... [31] The ligamento-muscular ... [32] Tissue adaptation to physical ... [33] The stabilizing system of ... [34] Phantom limb pain: A case ... [35] Functional outcome of lowerlimb ... [36] Three-dimensional motions of trunk ... [37] Transfemoral amputations: the effect ... [38] Energy storage and return prostheses ... [39] Gait comparisons for belowknee ... [40] Biomechanical significance of the correct length of lower limb prostheses ... [41] Low-back pain in transfemoral amputees ... [42] The influence of the length of lowerlimb prosthesis on spinal ... [43] Psychosocial factors and adjustment to chronic pain in persons with physical ... 




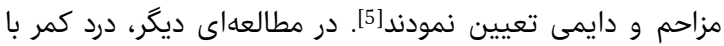





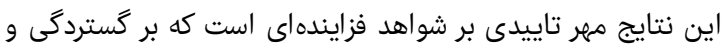

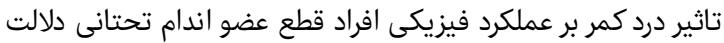

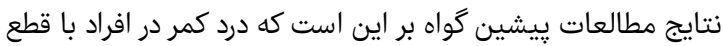

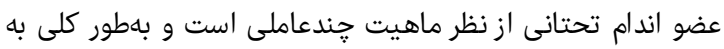







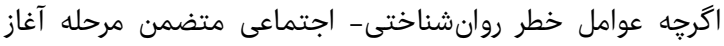







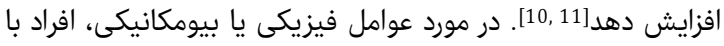

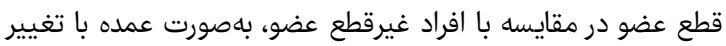

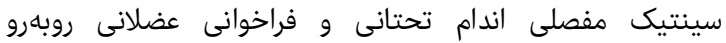



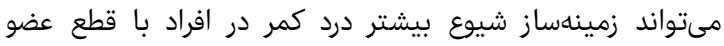

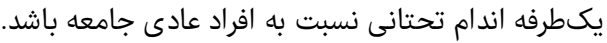



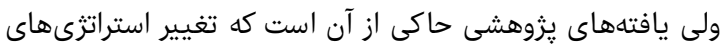









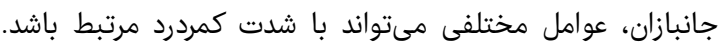

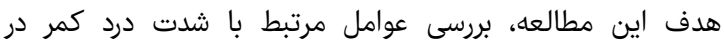

جانبازان قطع عضو يككرفه زير زانو مبتلا به كمردرد مزمن بود درد.

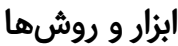

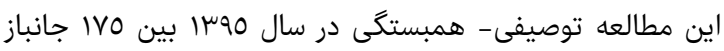

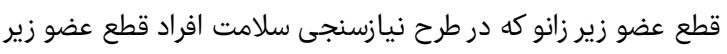

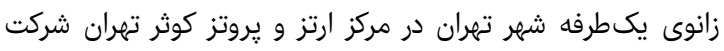











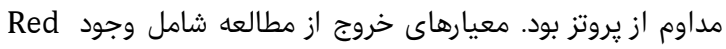

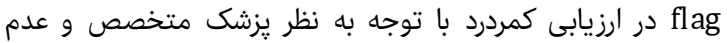

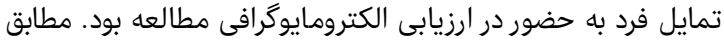

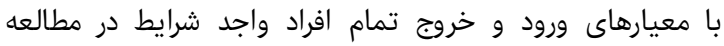
شركت كردند. ارزيابىهاى دموكرافيك و اختام اختلالات همراه باه با استفاده

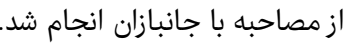
براى بررسى شدت درد كمر و عوامل مرتبط بان با آن ارزيابىهاى زير

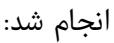
الف- ارزيابى شدت درد كمر و ناتوانى ناشى از درد كمر: از مقياس



عوامل مرتبط با شدت درد كمر در جانبازان قطع عضو يكنرفه زير زانو

الهه فرجى PhD كروه آسيبشناسى و حركات آرات اصلاحى، دانشكده تربيتبدنى و علوم ورزشى، إئى



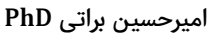
كروه آسيبشناسى و وراتى حركات اصلاحى، دانشكده تربيتبدنى و علوم ورزشى، إنى



حيدر صادقى PhD

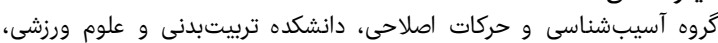





كروه طب فيزيكى و توانبخشى، دانشكده يزشكى، دانشكاه تهران، تهران، ايران BSc "مصطفى علامى فرونى

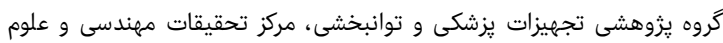
يزشكى جانبازان، تهران، ايران تريزي

קكيده اهّداف: كمردرد بهعنوان يك مشكل شايع، بيش از ساير عوارض قطع عضو







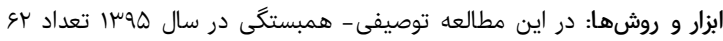

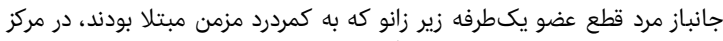











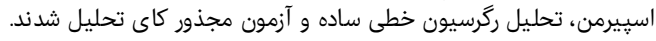



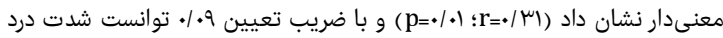







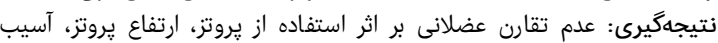



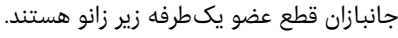
كليدوازهها: درد كمر، جانبازان، قطع عضوف الكترومايوزكرافى





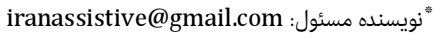



درد كمر يك مشكل شايع در عموم افراد يك جمعيت است و از



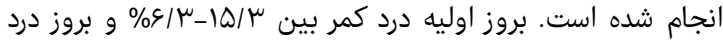

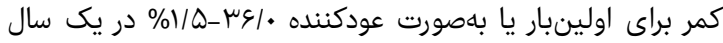

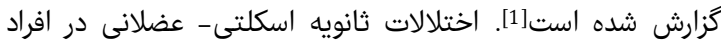



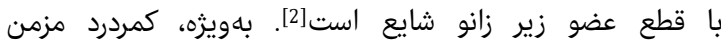












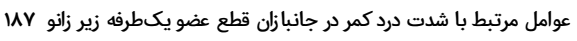

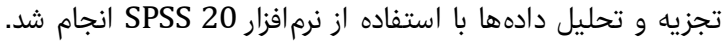

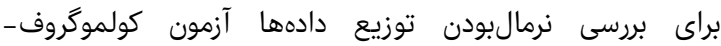

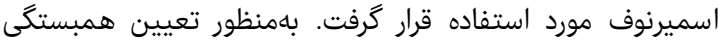

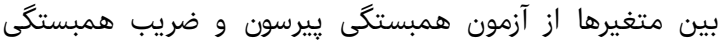

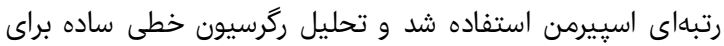

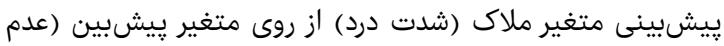

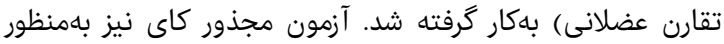

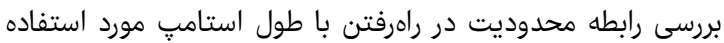
قرار گرفت

يافتهها

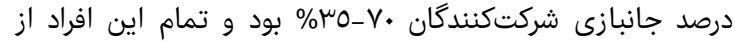

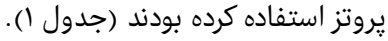

جدول () ميانكين آمارى متغيرهاى دموكرافيك جانبازان قطع عضو زير زانو

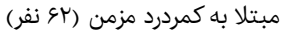

\begin{tabular}{|c|c|}
\hline ميانكين & متغيرها \\
\hline $0 . / 7 \pm 1 . / V$ & سن (سال) \\
\hline$r V / \mu \pm \mu / V$ & شاخص توده بدنى (كيلوكرم بر متر مربع) \\
\hline $\mathrm{rN} / \mathrm{Q}^{\prime} \mathrm{T} / \mathrm{V}$ & مدت سيرى شده از زمان قطع عضو (سال) \\
\hline$r N / V \pm T / q$ & دوره زمانى استفاده از يروتز (سال) \\
\hline $1 r / 7 \pm r / 9$ & استفاده روزانه از يروتز (ساعت) \\
\hline
\end{tabular}





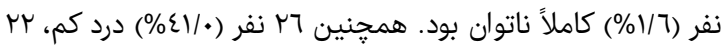

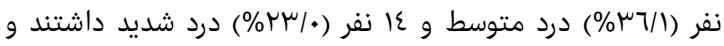

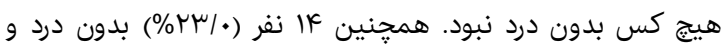

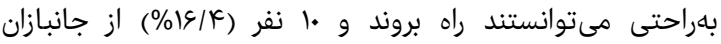

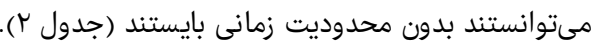

جدول r ميانكين آمارى نمرات شاخص ناتوانى، شدت درد كمر و ضريب عدم زئر

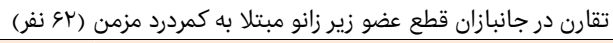

\begin{tabular}{|c|c|}
\hline نمرات & متغيرها \\
\hline & شاخص ناتوانى اوسوسترى \\
\hline \multirow{2}{*}{. } & نمره ناتوانى \\
\hline & شدت درد \\
\hline$r / 97 \pm 1 / V$. & در بهترين حالت \\
\hline$\varepsilon / 1 . \pm r / 0$. & در زمان مطالعه \\
\hline$V / \Delta F \pm 1 / \Lambda$. & در بدترين حالت \\
\hline 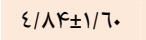 & شدت درد كلى \\
\hline & ضريب عدم تقارن \\
\hline$r \tau / \mu \cdot \pm r \mu / l$. & عضله اركتور اسياينه \\
\hline$r \varepsilon / r \cdot \pm I V / V$. & عضله مولتيىفيدوس \\
\hline
\end{tabular}

ضريب عدم تقارن عضله مولتىفيدوس با ساعات استفاده از يروتز

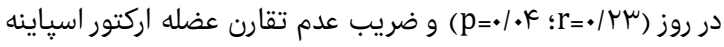

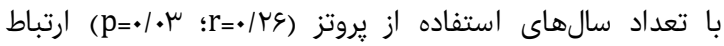

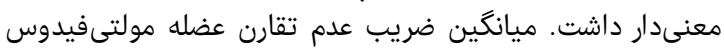

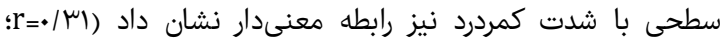

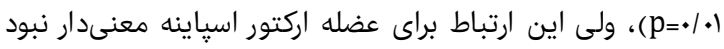

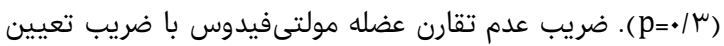

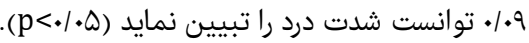

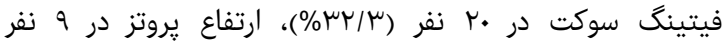

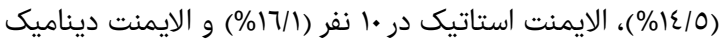

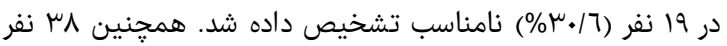

درجهبندى صفر تا •ا استفاده شد. شدت درد در زمان مطالعه،

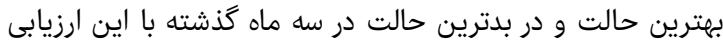

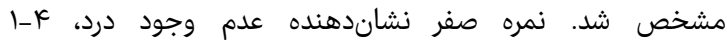

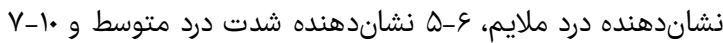

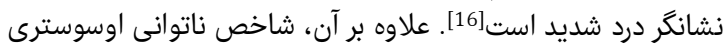

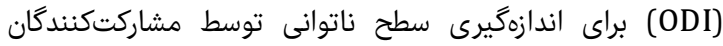

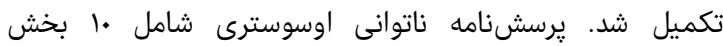

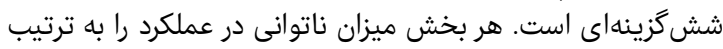

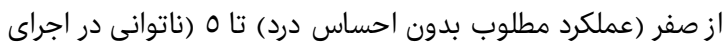

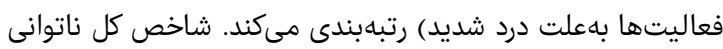

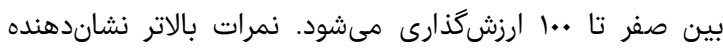

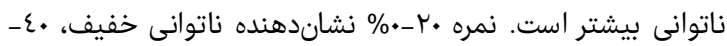

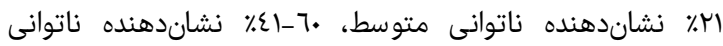

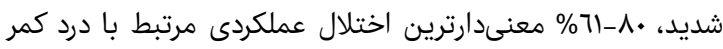

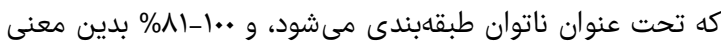

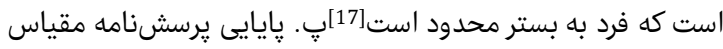

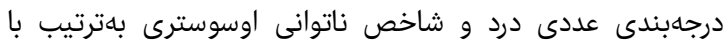

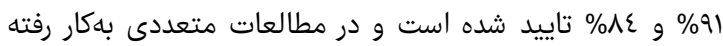

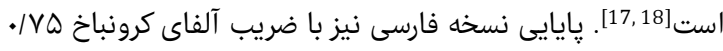

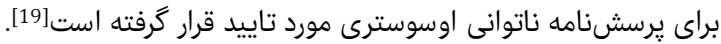

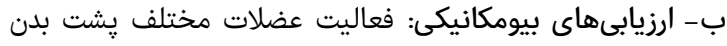

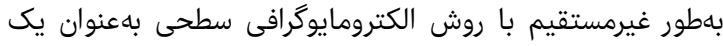





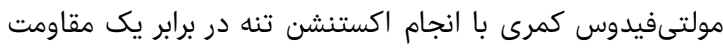

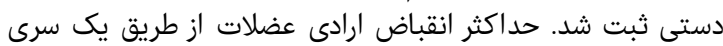

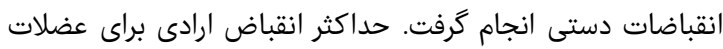

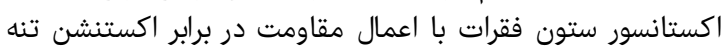

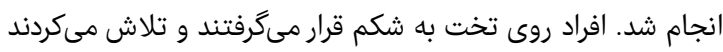



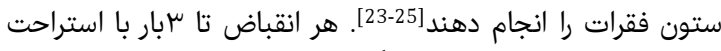

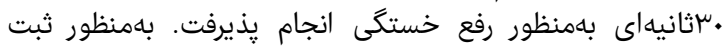

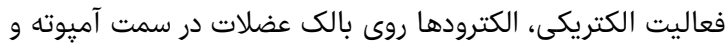

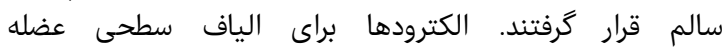

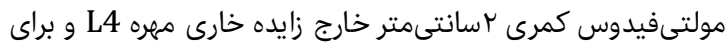
اركتور اسياينه كمرى هسانتىمتر خارج زايده خارى مهرى مهره L2 قرار

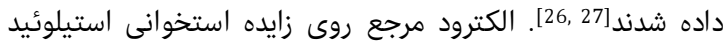

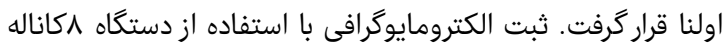
EMG

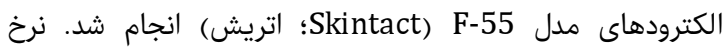

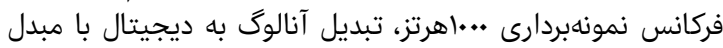
\& A/D

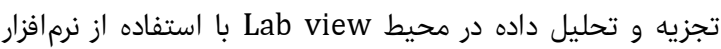
تحليل داده BayaMed انجام شد. ريشه ميانكين مربع (RMS)

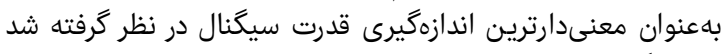

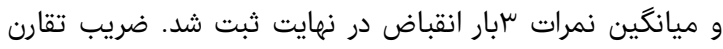

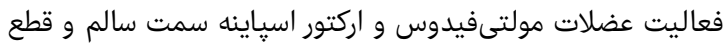

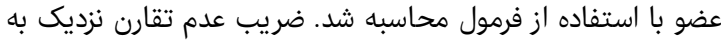

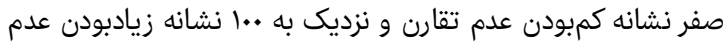

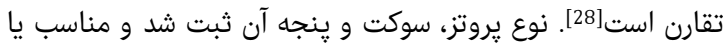

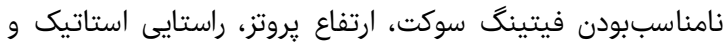
ديناميك يروتز توسط كارشناسها مورد ارزيابى بالينى قرار گرفتئ. 
جبرانى مىتواند يك علت احتمالى در ابتلا به درد كمر در افراد قطع

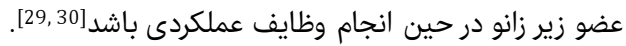

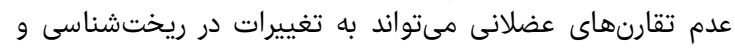

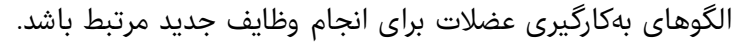

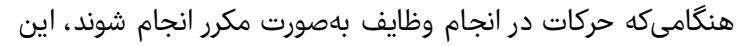

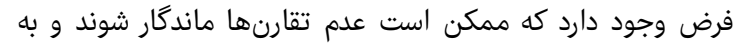

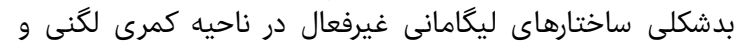

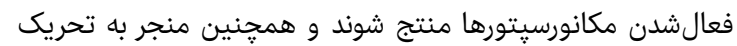

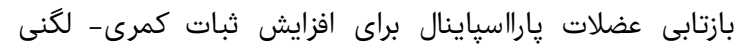

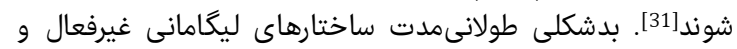

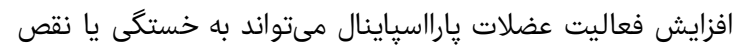

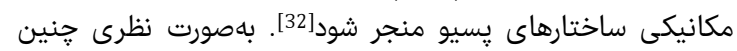

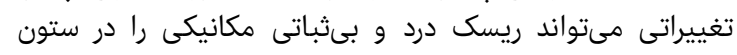

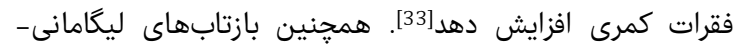

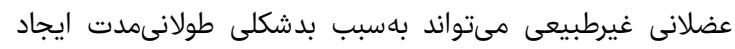

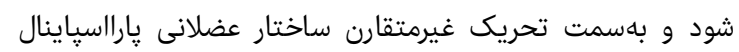

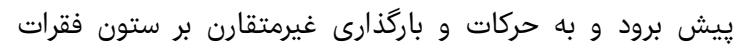

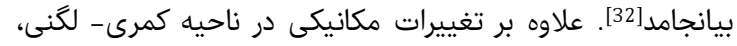

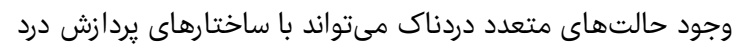

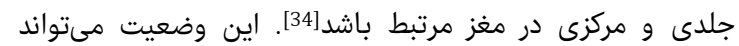

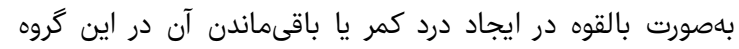

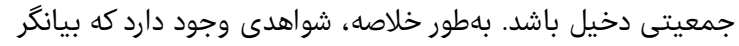

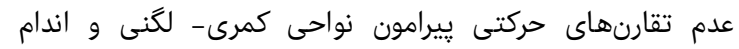

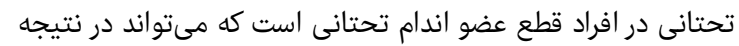

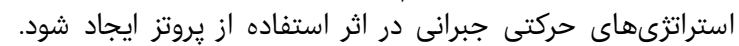

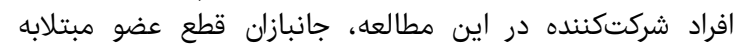

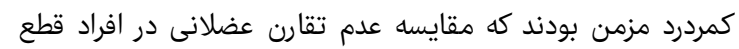

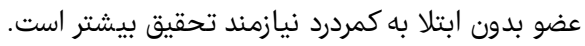

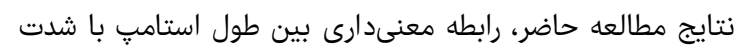

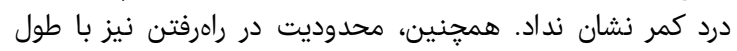

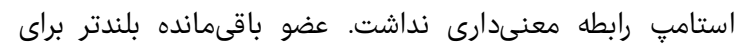

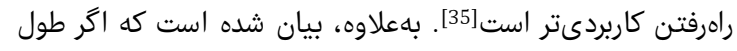

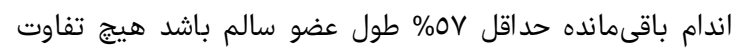

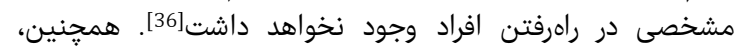

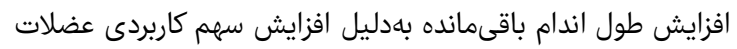

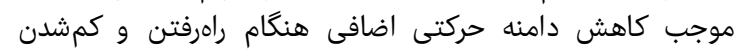

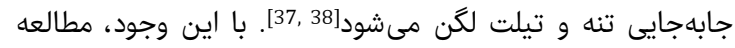

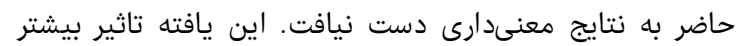

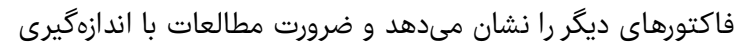

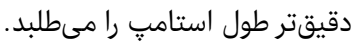

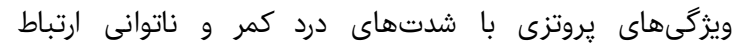

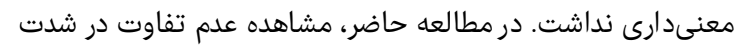

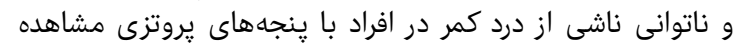

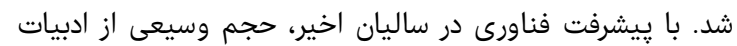

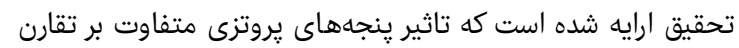

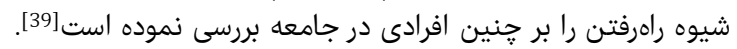

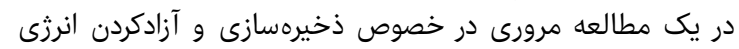

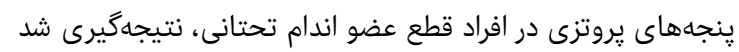

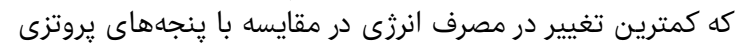

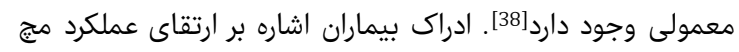

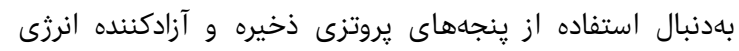

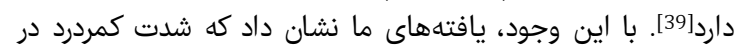

(\%7./V)

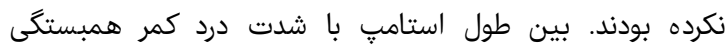

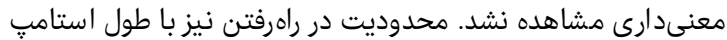

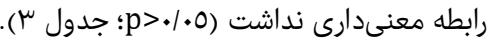

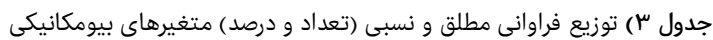

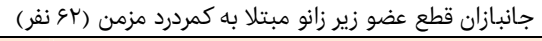

\begin{tabular}{|c|c|}
\hline تعداد (درصد) & متغير \\
\hline$(V \cdot / q) \varepsilon \varepsilon$ & نوع يروتز \\
\hline (P4/I) & يديلنى \\
\hline ( & لاينر \\
\hline ד & سافت - سوكت \\
\hline & نوع ينجه \\
\hline r & تكمحوره \\
\hline (T/I) & ساج \\
\hline$(10 / \wedge) 1$. & ديناميك \\
\hline$(10 / \Lambda) 1$. & كربنى \\
\hline$(9 / 7) 7$ & ساير \\
\hline & طول استامٍ \\
\hline سا & بسيار بلند \\
\hline$(1 N / 7) \mathbb{I r}$ & بلند \\
\hline ( & متوسط \\
\hline$(\mid \mu / 7) \wedge$ & كوتاه \\
\hline$(0 / 1)^{\mu}$ & بسيار كوتاه \\
\hline
\end{tabular}

سن و شاخص توده بدنى با شدت كمردرد همبستگى معنىدارى

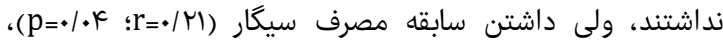

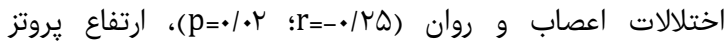

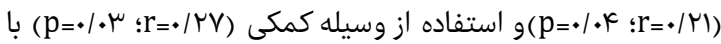

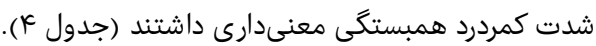

جدول ع) توزيع فراوانى مطلق و نسبى (تعداد و درصد) و ميانكين آمارى نمرات

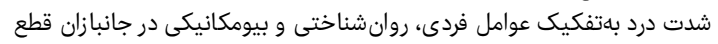

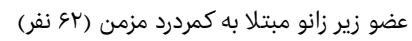

\begin{tabular}{|c|c|c|c|c|}
\hline نمره شدت درد & درد شديد & درد متوسط & درد كم & متغيرها \\
\hline & & & & سابقه مصرف سيكار \\
\hline $0 / \varepsilon \pm 1 / V$ & $(I r / 9) \wedge$ & $(11 / r) V$ & $(\Lambda / \cdot) 0$ & دارد \\
\hline \multirow[t]{2}{*}{$\varepsilon / 7 \pm 1 / 0$} & $(9 / 7) 7$ & $(Y O / \Lambda) 17$ & $(\mu r / r) r$. & ندارد \\
\hline & & & & آسيب اعصاب و روان \\
\hline$\Delta / \mu_{ \pm} \pm / Q$ & $(I r / 9) \wedge$ & $(19 / \mu)(r$ & $(1 r / 9) \wedge$ & دارد \\
\hline \multirow[t]{2}{*}{$Q / F \pm 1 / V$} & $(9 / 7) 7$ & $(I V / V) \|$ & $(Y V / F) I V$ & 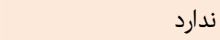 \\
\hline & & & & ارتفاع يروتز \\
\hline$\kappa / V \pm 1 / \varepsilon$ & $(I V / V) \|$ & $(r q / \cdot) \backslash \Lambda$ & س & مناسب \\
\hline \multirow[t]{2}{*}{$\Delta / \& \pm 1 / \mathbb{R}$} & $(\varepsilon / \Lambda)^{\mu}$ & $(\Lambda / \cdot) 0$ & $(\mu / r) r$ & ن نامناسب \\
\hline & & & & استفاده از وسيله كمكى \\
\hline$\Delta / \mathcal{F}_{ \pm 1 / \varphi}$ & $(I r / 9) \wedge$ & $(I r / 9) \wedge$ & $(1 Y / 9) \wedge$ & دارد \\
\hline$\varphi / \Delta \pm \mid / \varphi$ & $(9 / 7) 7$ & $(Y \varepsilon / 1) 10$ & $(Y V / F) I V$ & 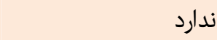 \\
\hline
\end{tabular}

بحث

در اين مطالعه، شدت درد كمر و ناتوانى ناشى از آن و همجنين

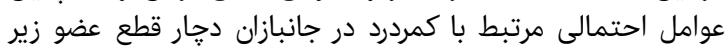

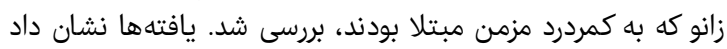

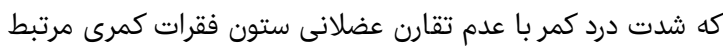

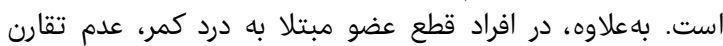

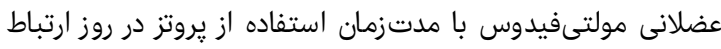
معنىدارى داشت. اين عدم تقارنهاى عضلانى همراه بان مان مكانيزم

فصل نامه علمى - يزووهشى طب جانباز 


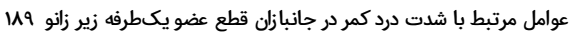

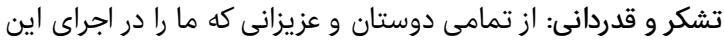

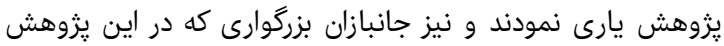

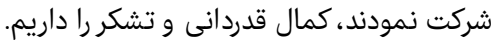

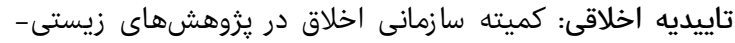

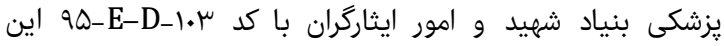

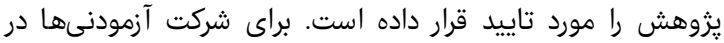

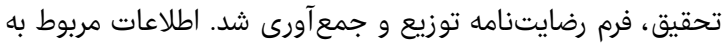

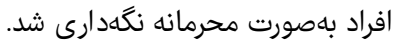

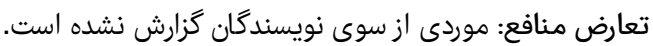

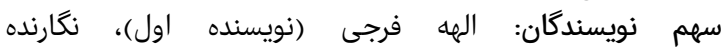

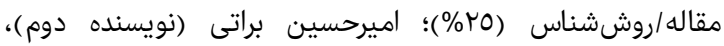

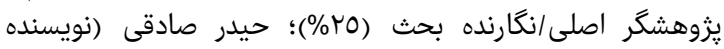

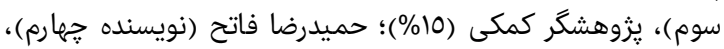

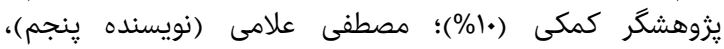

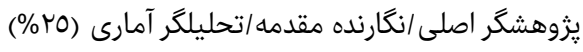

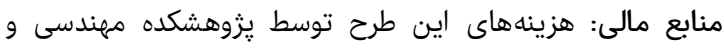

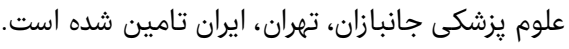

منابع 1- Hoy D, Brooks P, Blyth F, Buchbinder R. The epidemiology of low back pain. Best Pract Res Clin Rheumatol. 2010;24(6):769-81.

2- Robert Gailey PhD P. Review of secondary physical conditions associated with lower-limb amputation and long-term prosthesis use. J Rehabil Res Dev. 2008;45(1):15-29.

3- Smith DG, Ehde DM, Legro MW, Reiber GE, del Aguila $\mathrm{M}$, Boone DA. Phantom limb, residual limb, and back pain after lower extremity amputations.Clin Orthop Relat Res. 1999;361:29-38.

4- Ephraim PL, Wegener ST, MacKenzie EJ, Dillingham TR, Pezzin LE. Phantom pain, residual limb pain, and back pain in amputees: Results of a national survey. Arch Phys Med Rehabi. 2005;86(10):1910-9.

5- Ehde DM, Smith DG, Czerniecki JM, Campbell KM, Malchow DM, Robinson LR. Back pain as a secondary disability in persons with lower limb amputations. Arch Phys Med Rehabi. 2001;82(6):731-4.

6- Jensen MP, Smith DG, Ehde DM, Robinsin LR. Pain site and the effects of amputation pain: further clarification of the meaning of mild, moderate, and severe pain. Pain. 2001;91(3):317-22.

7- da Costa BR, Vieira ER. Risk factors for work-related musculoskeletal disorders: a systematic review of recent longitudinal studies. Am J Ind Med. 2010;53(3):285-323.

8- Devan H, Carman AB, Hendrick PA, Ribeiro DC, Hale LA. Perceptions of low back pain in people with lower limb amputation: A focus group study. Disabil Rehabil. 2015;37(10):873-83.

9- Koes B, Van Tulder M, Thomas S. Diagnosis and treatment of low back pain. BMJ. 2006;332(7555):1430-4. 10- Hoogendoorn WE, van Poppel MN, Bongers PM, Koes BW, Bouter LM. Systematic review of psychosocial factors at work and private life as risk factors for back pain. Spine. 2000;25(16):2114-25.

11- Pincus T, Burton AK, Vogel S, Field AP. A systematic review of psychological factors as predictors of chronicity/disability in prospective cohorts of low back pain. Spine. 2002;27(5): 109-20.

12- Silverman AK, Fey NP, Portillo A, Walden JG, Bosker G, Neptune RR. Compensatory mechanisms in below-
افراد با ينجههاى مختلف متفاوت نبود. با توجه به معنىداربودن

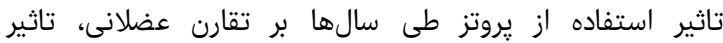

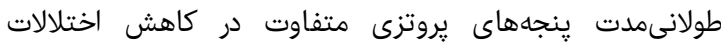
اسكلتى- عضلانى در افراد قطع عضو اندام تحتانى و و كمردرد

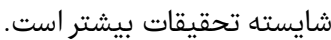

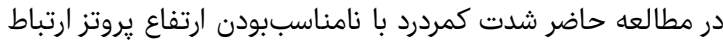

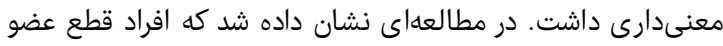

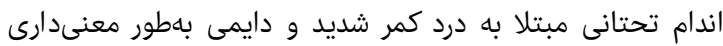

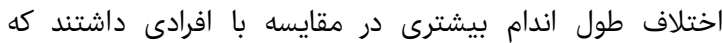

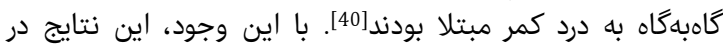

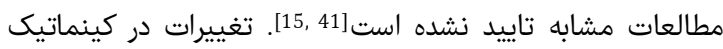

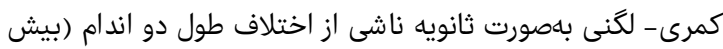

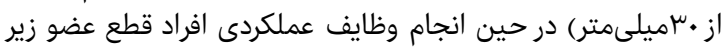

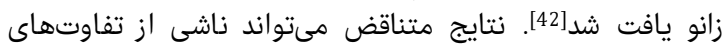

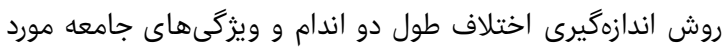

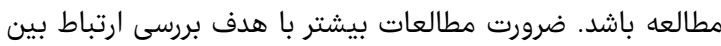

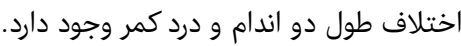

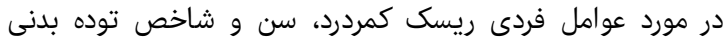

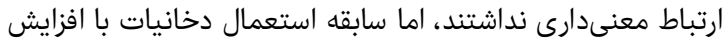

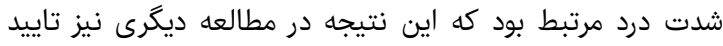

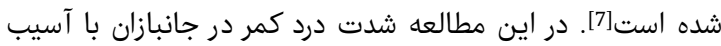

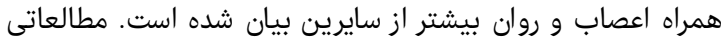

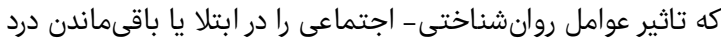

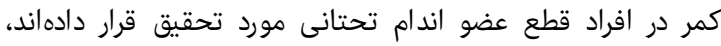

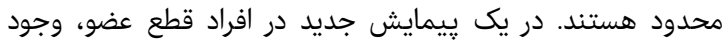

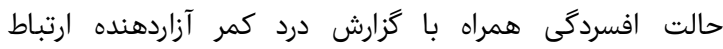

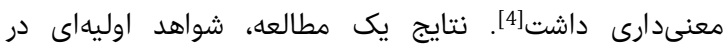
خصوص وجود ارتباطى بين عوامل روان شناختى - اجتماعى (مانند

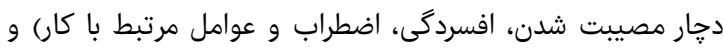

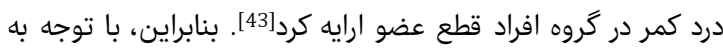

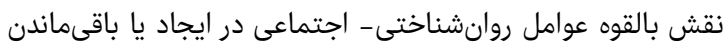

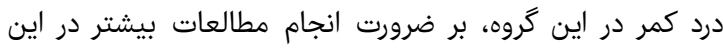
خصوص دلالت مىكند.

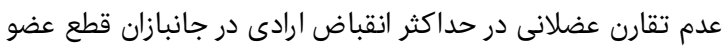

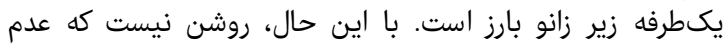

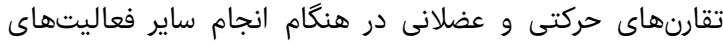

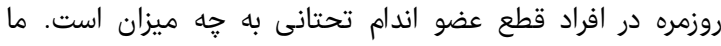

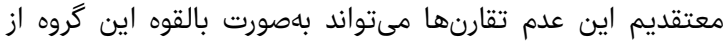

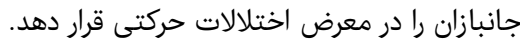

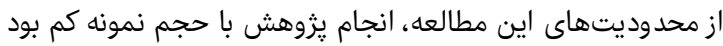

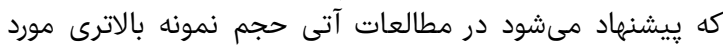
براسى قرار گيرد.

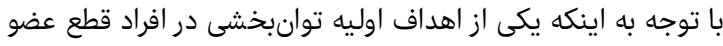

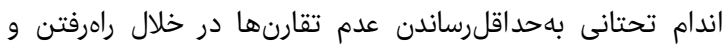

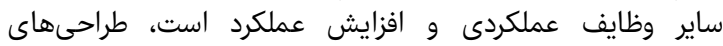

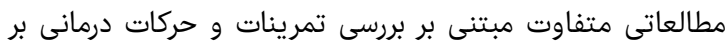

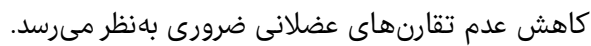

\section{نتيجهيرى}

عدم تقارن عضلانى بر اثر استفاده از يروتز، ارتفاع ڤِروتز، آسيب

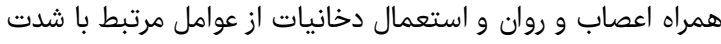
درد كمر در جانبازان قطع عضو يكسطرفه زير زانو هستند. 
neuromuscular training on non-dominant side of low back improves balanced muscle response and spinal stability. Eur J Appl Physiol . 2013;113(4):997-1004.

29- Michaud SB, Gard SA, Childress DS. A preliminary investigation of pelvic obliquity patterns during gait in persons with transtibial and transfemoral amputation. J Rehabil Res Dev. 2000;37(1):1-10.

30- Prinsen EC, Nederhand MJ, Rietman JS. Adaptation strategies of the lower extremities of patients with a transtibial or transfemoral amputation during level walking: a systematic review. Arch Phys Med Rehabil. 2011;92(8):1311-25.

31- Solomonow M, Zhou B-H, Harris M, Lu Y, Baratta RV. The ligamento-muscular stabilizing system of the spine. Spine. 1998;23(23):2552-62.

32- Mueller MJ, Maluf KS. Tissue adaptation to physical stress: A proposed "Physical stress theory" to guide physical therapist practice, education, and research. Physical therapy. 2002;82(4):383.

33- Panjabi MM. The stabilizing system of the spine. part II. neutral zone and instability hypothesis. J Spinal Disord. 1992;5(4):390-7.

34- Flor H, Nikolajsen L, Jensen TS. Phantom limb pain: A case of maladaptive CNS plasticity?. Nat Rev Neurosci. 2006;7(11):873-81.

35- Greive AC, Lankhorst GJ. Functional outcome of lower-limb amputees: A prospective descriptive study in a general hospital. Prosthet Orthot Int. 1996;20(2):79-87. 36- Goujon-Pillet H, Sapin E, Fodé P, Lavaste F. Threedimensional motions of trunk and pelvis during transfemoral amputee gait. Arch Phys Med Rehabil. 2008;89(1):87-94.

37- Bell JC, Wolf EJ, Schnall BL, Tis JE, Tis LL, Potter MBK. Transfemoral amputations: the effect of residual limb length and orientation on gait analysis outcome measures. J Bone Joint Surg Am. 2013;95(5):408-14.

38- Hafner BJ, Sanders JE, Czerniecki J, Fergason J. Energy storage and return prostheses: does patient perception correlate with biomechanical analysis? Clin Biomech. 2002;17(5):325-44.

39- Macfarlane PA, Nielsen DH, Shurr DG, Meier K. Gait comparisons for below-knee amputees using a flex-foot versus a conventional prosthetic foot. J Prosthet Orthot. 1991;3(4):150-61.

40- Friberg 0 . Biomechanical significance of the correct length of lower limb prostheses: a clinical and radiological study. Prosthet Orthot Int. 1984;8(3):124-9. 41- Morgenroth DC, Shakir A, Orendurff MS, Czerniecki JM. Low-back pain in transfemoral amputees: is there a correlation with static or dynamic leg-length discrepancy?. Am J Phys Med Rehabil. 2009;88(2):10813.

42- Lee RY, Turner-Smith A. The influence of the length of lower-limb prosthesis on spinal kinematics. Arch Phys Med Rehabil. 2003;84(9):1357-62.

43- Jensen MP, Moore MR, Bockow TB, Ehde DM, Engel JM. Psychosocial factors and adjustment to chronic pain in persons with physical disabilities: A systematic review. Arch Phys Med Rehabil. 2011;92(1):146-60. knee amputee gait in response to increasing steady-state walking speeds. Gait Posture. 2008;28(4):602-9.

13- Silverman AK, Neptune RR. Three-dimensional knee joint contact forces during walking in unilateral transtibial amputees. J Biomech . 2014;47(11):2556-62.

14- Fey NP, Silverman A, Neptune R. The influence of increasing steady-state walking speed on muscle activity in below-knee amputees. J Electromyogr Kinesiol. 2010;20(1):155-61.

15- Kulkarni J, Gaine W, Buckley J, Rankine J, Adams J. Chronic low back pain in traumatic lower limb amputees. Clin Rehabil. 2005;19(1):81-6.

16- Serlin RC, Mendoza TR, Nakamura Y, Edwards KR, Cleeland CS. When is cancer pain mild, moderate or severe? Grading pain severity by its interference with function. Pain. 1995;61(2):277-84.

17- Fairbank J, Couper J, Davies J, O'brien J. The Oswestry low back pain disability questionnaire. Physiotherapy. 1980;66(8):271-3.

18- Price DD, McGrath PA, Rafii A, Buckingham B. The validation of visual analogue scales as ratio scale measures for chronic and experimental pain. Pain. 1983;17(1):45-56.

19- Mousavi SJ, Parnianpour M, Mehdian H, Montazeri A, Mobini B. The Oswestry disability index, the RolandMorris disability questionnaire, and the Quebec back pain disability scale: Translation and validation studies of the Iranian versions. Spine. 2006;31(14):454-9.

20- De Luca CJ. Use of the surface EMG signal for performance evaluation of back muscles. Muscle Nerve. 1993;16(2):210-6.

21- Kramer M, Ebert V, Kinzl L, Dehner C, Elbel M, Hartwig E. Surface electromyography of the paravertebral muscles in patients with chronic low back pain. Arch Phys Med Rehabil. 2005;86(1):31-6.

22- Hu Y, Siu SH, Mak JN, Luk KD. Lumbar muscle electromyographic dynamic topography during flexionextension. J Electromyogr Kinesiol. 2010;20(2):246-55.

23- Moffroid MT. Endurance of trunk muscles in persons with chronic low back pain: assessment, performance, training. J Rehabil Res Dev. 1997;34(4):440-7.

24- Drake JD, Fischer SL, Brown SH, Callaghan JP. Do exercise balls provide a training advantage for trunk extensor exercises? A biomechanical evaluation. J Manipulative Physiol Ther. 2006;29(5):354-62.

25- McGill SM. Electromyographic activity of the abdominal and low back musculature during the generation of isometric and dynamic axial trunk torque: Implications for lumbar mechanics. J Orthop Res. 1991;9(1):91-103.

26- Claus AP, Hides JA, Moseley GL, Hodges PW. Different ways to balance the spine: subtle changes in sagittal spinal curves affect regional muscle activity. Spine. 2009;34(6):E208-14.

27- Tsao H, Druitt TR, Schollum TM, Hodges PW. Motor training of the lumbar paraspinal muscles induces immediate changes in motor coordination in patients with recurrent low back pain. Pain. 2010;11(11):1120-8. 28- Kim Y, Son J, Yoon B. Intensive unilateral 